\title{
CLASES SOCIALES E IDENTIDAD PERSONAL: ESTUDIO COMPARATIVO EN ADOLESCENTES ESCOLARIZADOS ${ }^{1}$
}

\author{
SOCIAL CLASSES AND EGO IDENTITY: \\ COMPARATIVE STUDY ON TEENAGERS AT HIGH SCHOOL
}

\section{Juan Diego García C.*}

\begin{abstract}
"Cuando un psicólogo, analiza la psicología de los individuos, el material con que se encuentra es la psicología de clases." Ignacio Martín-Baro, Acción e Ideología, 1983/2004
\end{abstract}

\section{RESUMEN}

El artículo da cuenta de una investigación que estudia la relación entre la condición de clase y las características de la identidad personal en 219 adolescentes, mujeres y hombres, de 16 y 17 años, de un colegio público y uno privado de San José. Se utilizó una metodología cuantitativa, transversal y correlacional con instrumentos sobre aspectos socioeconómicos, uso del tiempo libre, identidad personal y estima de sí. Los resultados apoyan la hipótesis de la incidencia de la clase social en la configuración de la subjetividad.

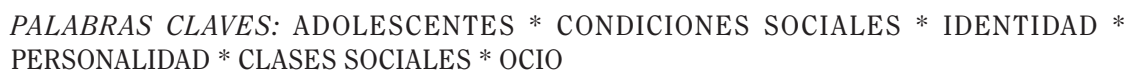

\section{ABSTRACT}

The article offers an investigation that examines the relationship between social class condition and the characteristics of ego identity in 219 adolescents, men and women, of 16 and 17 years old, from a public and a private high school in San Jose. The methodology was quantitative, transversal and correlational with instruments of socioeconomic aspects, ego identity, self-esteem and free time. The results support the hypothesis of the incidence of social class in the configuration of subjectivity.

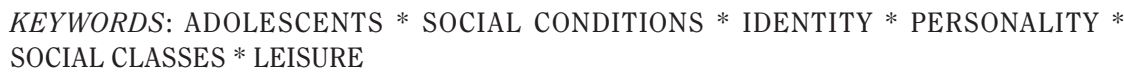

El artículo presenta los resultados generales de la investigación que el autor realizó como tesis de licenciatura en psicología de la Universidad de Costa Rica. Contó con el apoyo académico del Instituto de Investigaciones Psicológicas y una beca del Instituto de Investigaciones Sociales.
Especial agradecimiento a Laura Sánchez, Keylin Rodríguez y Ernesto Herra por sus comentarios a este artículo.

* Instituto de Investigaciones Psicológicas, Universidad de Costa Rica. juandiego48@gmail.com 


\section{INTRODUCCIÓN}

La realidad costarricense es cada vez más compleja y polarizada. El país se debate entre diferentes modelos de desarrollo que responden a las clases sociales que luchan entre sí. En estos espacios, los adolescentes van construyendo una identidad personal que les permite enfrentarse a la vida $y$ a su espacio social. No hay una Costa Rica igual para todos ni por ende un único tipo de adolescencia.

La investigación tiene como premisa conocer cuál es la relación entre las clases sociales y las características de la identidad personal en adolescentes escolarizados de San José.

Según el Informe del Estado de La Nación (2007), a pesar que la economía del país se fortalece, la desigualdad en la distribución de los ingresos sigue creciendo y la pobreza no disminuye. $\mathrm{El} \mathrm{20 \%} \mathrm{de} \mathrm{la} \mathrm{población} \mathrm{con} \mathrm{mayor}$ ingreso recibe 10,2 veces más que el $20 \%$ de la población con menor ingreso. El segmento más bajo gasta mensualmente menos de 40000 colones per capita frente a los 450000 colones per capita que consume el segmento más alto.

En términos educativos, las diferencias entre los colegios públicos y privados son considerables. Un estudiante de un colegio público recibe 3 veces menos dinero que un estudiante de un colegio privado, $y$ su familia en general recibe 4 veces menos. En promoción, se gradúan 20\% más los estudiantes en educación privada. En deserción, en la educación pública un $10 \%$ más estudiantes abandonan las aulas. El principal motivo de inasistencia y deserción sigue siendo a través de los años el no poder pagar los estudios (Estado de la Nación, 2006).

El tema no es simple preocupación conceptual, es una realidad que abofetea la cara. La disparidad es reflejo de una sociedad en la cual las prácticas cotidianas transcurren en espacios sociales cada vez menos comunes.

El estudio compara cómo el contexto de desarrollo del adolescente influye en su construcción del sí mismo y su identidad personal. Para ello, se trabajó con la totalidad de la población de cuarto y quinto año del colegio público ubicado en la zona de mayor rezago social de San José y uno de los colegios privados más caros del país.
El tema de la identidad personal en adolescentes es una de las principales áreas de investigación en la psicología contemporánea (Schwartz, 2001). Sin embargo, en nuestro país $y$ en América Latina no ha sido un tema prioritario y cuenta con pocas investigaciones empíricas que lo sustenten. Por otro lado, el concepto de clase social es polémico por razones ideológicas y de profunda complejidad.

La relación entre macroestructura y subjetividad es un tema de largo debate en las ciencias sociales. Su estudio en Costa Rica busca desentrañar cómo el sistema de producción capitalista moldea quiénes somos, cómo nos vemos y qué deseamos hacer.

El artículo presenta en primera instancia los principales antecedentes empíricos, luego desarrolla el marco teórico de referencia, explica la metodología y finaliza mostrando y discutiendo sus principales hallazgos.

\section{ANTECEDENTES}

En Costa Rica no se han realizado investigaciones de este tipo, se ha estudiado la identidad personal en adolescentes pero no comparándolos por clase social. De hecho, el análisis de las características psicológicas de las clases sociales no es común. Sin embargo, hay tres investigaciones que son importantes para el objeto en estudio.

Quince años antes, Alarcón y Rosabal (1993) en un estudio sobre conciencia moral e identidad del yo compararon adolescentes de un colegio público y uno privado y concluyeron que: los jóvenes de colegios públicos están más orientados a la colectividad en contraste con los jóvenes de colegios privados que están más orientados a la individualidad.

Los adolescentes de colegios públicos son más críticos, establecen relaciones más horizontales, admiran más a la madre y se identifican más entre sí mientras que los jóvenes de colegios privados son menos críticos, establecen relaciones verticales, admiran más al padre y se identifican más con las figuras de autoridad que con su grupo de pares. Aunado a esto, encontraron que los jóvenes de colegios públicos identifican 
otras clases sociales y sienten enojo contra ellas y que los jóvenes de colegios privados no (Alarcón y Rosabal, 1993).

En otras latitudes, James Coté ha sido el investigador que más atención le ha brindado a las implicaciones de la estructura económicacultural en la construcción de la identidad personal (Schwartz, 2001).

Como parte de sus resultados de investigaciones en Canadá, Coté (1996) señala que los jóvenes varones de clases sociales bajas adquieren primero independencia de su familia $y$ desarrollo de identidad capital, entendiéndose esta como quién se es y qué se ha logrado. Este desarrollo en las clases bajas se explica por la necesidad de definir más temprano qué se va hacer para vivir.

En el seguimiento de la misma investigación, Coté (2002) encuentra que los jóvenes varones de clases altas cuentan con menos recursos identitarios en la mediana edad que los jóvenes varones de clases bajas. Esto debido a la menor necesidad durante el desarrollo de su ciclo vital de resolver más problemas por ellos mismos, ya que siempre cuentan con el respaldo económico y social de sus familias.

Caso contrario al de los jóvenes de clase trabajadora quiénes por sus condiciones socioeconómicas tienen que resolver solos más tareas del desarrollo desde una edad más temprana. De esta forma, los jóvenes de familias de clase trabajadora cuentan con un mayor desarrollo de identidad capital y con más recursos identitarios a mediano plazo que los jóvenes burgueses (Coté, 2002). Como se verá más adelante, hay coincidencia entre esos datos con los obtenidos en el país.

\section{LA CLASE SOCIAL COMO ELEMENTO CONTEXTUAL DE LA IDENTIDAD PERSONAL}

El concepto de clases sociales es parte de una teoría y corriente de pensamiento que no sólo intenta describir la realidad social, sino explicar su funcionamiento y transformación (Cardoso y Pérez, 1982). En los últimos años, se ha usado poco principalmente por la presión ideológica de los centros imperiales, y por la distancia de los académicos e intelectuales con las clases populares (Lott, 2002).

Las clases sociales son grupos reales de personas que ocupan lugares distintos en la producción social. El lugar que ocupan depende de la relación con los medios de producción, por su papel en la organización social del trabajo y por el volumen de riqueza social con el que cuentan. Gracias a esta diferenciación, un grupo de personas (una clase social) puede dejarse el trabajo de otros, es decir, explotarlos (Antunes, 2000).

El modo de producción es la manera específica de una sociedad para producir los bienes materiales que necesita. Tiene un carácter específico, histórico y transitorio. Caracteriza la relación del ser humano con la naturaleza a través del trabajo: con el medio para desarrollarlo $y$ con el objeto producto de estos.

Las personas dentro del capitalismo se relacionan en dos formas fundamentales con los medios de producción: quiénes son los dueños de los medios y propietarios de sus productos (clase burguesa) y quiénes no tienen medios de producción y venden a los burgueses su fuerza de trabajo a cambio de un salario (clase proletaria). El burgués devuelve al trabajador una parte de lo que este produce en el salario, pero se deja el excedente, la plusvalía. De esta forma arranca al trabajador parte de su trabajo y se apropia de él (Antunes, 2000).

Las clases sociales solo existen enfrentadas entre sí. La realidad de la clase social, así como la pertenencia de un individuo a ella, son hechos objetivos que no dependen de la conciencia ni de la voluntad subjetiva de las personas (Martín-Baró, 1983/2004).

La explotación burguesa no se llevaría a cabo sin el control de los medios de socialización, grupos e instituciones sociales que toman parte en el control ideológico y mental de la clase trabajadora. Por ejemplo los periódicos, la radio, la televisión, el cine, el teatro, las iglesias, los partidos políticos, las escuelas, los intelectuales, el estado y por supuesto el sistema educativo. Estos ayudan a la burguesía a garantizar sus privilegios (Miliban, 1991).

De hecho, esta investigación se interesa en el sistema educativo por ser parte central del 
espacio social a través del cual los adolescentes construyen su identidad personal. El espacio social es la división de grupos de personas a través de diferencias observadas en su realidad cotidiana (Bourdieu, 2000). La estructura de clases deja su impronta en el adolescente. No es lo mismo una familia donde el papá sale a trabajar de guarda que aquella donde el papá profesional es dueño de su propio negocio $y$ contrata a ese guarda para que se lo cuide. El tipo de trabajo que se realiza incide en la identidad personal y por ende, en la crianza y socialización de los hijos (Frable, 1997).

Es aquí donde la clase social se convierte en un elemento constitutivo de la identidad personal en los adolescentes, no sólo por el trabajo que realizan los padres sino por el espacio social donde se desenvuelven los hijos. ¿Por qué?

Porque la mediación entre el capital de los padres y sus hijos hace que estos participen de determinados espacios sociales característicos de su clase, por ejemplo el tipo de colegio al que asisten. En la adolescencia, el colegio es uno de los elementos más importantes del espacio social, y a su vez, el colegio es el intermediario entre la clase social de la familia y el adolescente.

Bourdieu (2000) explica como con el tiempo de compartir en un determinado espacio social se van adquiriendo sus prácticas de manera inconsciente como habitus. De esta forma, la identidad personal y la estima de sí particularizan el espacio social porque estas son reflejo del lugar en la sociedad que ocupa el adolescente.

La formación de la identidad personal es uno de los eventos más importantes en el desarrollo de la personalidad. Envuelve una síntesis de la experiencia infantil, dirigida a un más o menos coherente conjunto único que provee al joven adulto de un sentido de continuidad con el pasado y una dirección para el futuro. Responde a lo que los otros dicen que el individuo es $y$, además, a lo que él dice ser (Marcia, 1993).

La socialización es el medio a través del cual las personas construyen su identidad personal. El yo es el resultado de la socialización en todas sus dimensiones, $y$ cada momento de su evolución representa la síntesis final de los procesos evolutivos de la persona (Martín-Baró, 1983/2004).

Marcia (2002), basado en el desarrollo psicosocial de formación de la identidad descrito por Erickson construyó un modelo socioafectivo y psicodinámico de entender el proceso de formación de la identidad personal. El ciclo vital del individuo se divide en períodos cronológicos, los cuales están marcados por una crisis del yo en la que se puede avanzar, quedarse estático o retroceder. Dentro de estos períodos, la adolescencia es importante porque integra una estructura que no es finita, pero que da al sujeto una constitución firme en su trayecto vital.

El proceso de construcción de la identidad consiste en tomar decisiones y comprometerse, un proceso que en el mejor de los casos es precedido por una exploración de alternativas. Entre estos dominios donde se explora, se decide y se compromete se encuentran: la ocupación, las creencias religiosas, la política, las prioridades familiares y las vocacionales, en lo que se denomina como dimensión societal. La dimensión interpersonal comprende prácticas sociales $y$ valores interpersonales $y$ sexuales (Marcia, 2002).

Marcia (1993) tomó dos de las principales dimensiones descritas por Ericsson (1972): exploración y compromiso, para desarrollar su modelo. La exploración se puede definir como una situación a resolver donde el individuo evalúa información de sí mismo y del ambiente. El compromiso representa la adherencia a un sistema específico de valores, metas y creencias. En la bifurcación de la exploración y el compromiso, entre altos $y$ bajos niveles, $y$ la yuxtaposición de cada nivel de uno con cada nivel del otro, se derivan cuatro posiciones de la identidad: difusión, forclusión, moratoria y realización. Cada posición representa una combinación de un nivel de exploración y compromiso.

La posición difusión se caracteriza por no tener una identidad definida. Hay poco interés en el futuro y las relaciones con los padres no son buenas. No hay un sentido central del yo, la suerte juega un papel importante y existe un sentimiento de no contar con ningún control sobre el propio futuro. No hay confianza. El locus interno de control, auto regulación, es bajo, el 
locus de control es externo. Hay niveles bajos de autonomía y altos de sumisión (Marcia, 1993).

En la forclusión se tienen proyectos, pero son de los padres o de las figuras de autoridad. Se intenta vivir ideales, proyectos $y$ formas interpersonales que han sido predispuestas. La estima de sí está construida en relación con las tareas que se han asignado. Hay confianza pero no hay exploración de alternativas. Bajo locus de control interno, auto regulación, locus de control es alterno. Tendencias al autoritarismo y al pensamiento estereotipado (Marcia, 1993).

La moratoria es una posición en transición entre el no tener un sentido de identidad hasta construirla. Existe apego a una parte del pasado, mientras se camina hacia el futuro generalmente con mucha vacilación, miedo, intensidad y excitabilidad. Todas las cosas parecen posibles. Mucha preocupación. El mundo fenomenológico es consumido por la resistencia. Vaga confianza y se están explorando alternativas. Locus de control interno aunque alterno y autorregulación (Marcia, 1993).

Realización es la última posición del proceso, la identidad ya se ha logrado construir. Proyectos propios, no son los de los padres y están sujetos a revisión. El futuro se ve como algo en construcción o planificado. Hay confianza y exploración de alternativas. Se posee un locus de control interno, auto regulación. Autonomía, menos conformismo con la autoestima y menor disconformidad que los individuos de otras posiciones (Marcia, 1993).

En síntesis, los adolescentes de la clase trabajadora y los adolescentes burgueses van construyendo su identidad personal en espacios sociales distintos. Espacios llenos de contradicciones y opuestos entre sí, los cuáles contextualizan la vida cotidiana de los jóvenes.

\section{METODOLOGÍA}

Se buscaron colegios opuestos que representaran las clases sociales del país. En el caso del colegio público con el censo del 2000 del INEC (2006), los lugares prioritarios del gobierno en el 2005 (MIDEPLAN, 2005) y el Estado de la Nación (2006) se escogió a un colegio público de
San Felipe de Alajuelita por ser un lugar dentro del área metropolitana con rezago social según el INEC (2006), un distrito prioritario según MIDEPLAN (2005) y el cantón con los índices más bajos de desarrollo dentro del área metropolitana según el Estado de la Nación (2006). Un año después de escoger el distrito y el cantón, el Estado de la Nación (2007) señaló a Alajuelita como uno de los principales cantones del área metropolitana con rezago generalizado, lo cual confirma y justifica el procedimiento utilizado en la escogencia de este colegio.

Para el colegio privado se contactó a uno de los centros educativos con la mensualidad más alta del país, y se trabajó con un colegio de Escazú donde la población de la muestra, pagaba en el momento de recolección de datos en el 2007, la cantidad de 348000 colones por mes, el equivalente a $\$ 690$, sin contar otros rubros como matrícula, transporte, uniformes o alimentación. Al igual que en el caso del colegio público, un año después de escoger el lugar, el Estado de la Nación (2007) ubica a Escazú como uno de los primeros cantones en polarización y desigualdad social. Lo cual también justifica la elección realizada.

La recolección de datos se realizó en un solo momento. Los estudiantes tenían que ser costarricenses. En el colegio privado, no podían recibir ayuda socioeconómica o beca y en el colegio público, debían pertenecer a la zona geográfica de incidencia del colegio.

Participaron 427 estudiantes en total (pruebas pilotos y recolección final de datos): mujeres $y$ hombres, con edades entre los 16 y 17

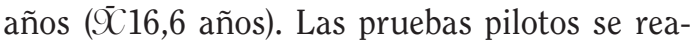
lizaron para analizar y mejorar las cualidades internas de los instrumentos, participaron 205 estudiantes con características similares a los de la recolección final de datos, en la que participaron 222 adolescentes.

Los instrumentos utilizados fueron: el Inventario de estilos de identidad personal (IEIPCR) (Berzonsky, 1992) el cuál se adaptó al país para esta investigación, la Escala de Posiciones de la Identidad (EPI-CR) (Adams, 1998), una escala de autoestima (Rosenberg, 1973; Smith, 2003), una escala de deseabilidad social (Castro, 2006), un índice socioeconómico (Pérez, 2006), 
un índice de tiempo libre (Pérez, 2006) y se pidió información sociodemográfica. Todos los instrumentos son escalas tipo Likert que fueron sistematizados $y$ analizados con el programa estadístico SPSS/15 (Programa Estadístico para Ciencias Sociales, por sus siglas en inglés).

Los resultados se contrastaron por clase social, género y entre cada una de las escalas. En el análisis se hizo uso de técnicas de estadística descriptiva e inferencial: análisis factorial, índice Alfa de Cronbach, la prueba de independencia entre variables chi-cuadrada $\left(\mathrm{X}^{2}\right)$, correlación lineal $r$ de Pearson y la comparación de medias ANOVA que es central en la investigación porque es la que indica si existe una relación entre clase social, género e identidad personal y cómo es esta relación.

\section{RESULTADOS}

Se presentan en primera instancia la comparación de medias por sexo de la dimensión interpersonal de la Escala de Posiciones de la Identidad (EPI/64-CR). La dimensión interpersonal comprende los dominios de: amistad, flirteo, roles sexuales y recreación y se caracteriza por la relación que el sujeto tiene con otras personas y con sí mismo a través de estos dominios (Adams, 1998). El EPI/64-CR procura establecer cuál es el momento en el proceso de formación de la identidad en la cual se encuentra el adolescente.
Las posiciones obtuvieron un coeficiente Alfa de Cronbach de: difusión .66, forclusión .78 , moratoria .53 y realización .58. Los índices con los que se trabaja a nivel internacional las posiciones de identidad van desde .55 a .76 con un promedio de .64 (Adams, 1998). Para el caso de moratoria el análisis se realiza con la precaución de su índice de confiabilidad.

En el cuadro 1 se observa que existen diferencias significativas en la posición de realización $(F(1,205)=6.022, p=.015$, con $\mathrm{M}=35.9$ para las mujeres y $\mathrm{M}=33.78$ para los hombres), en donde las mujeres tienden a poseer promedios mayores con respecto a los hombres.

En el cuadro 2 se observa que hay diferencias significativas por colegio a nivel de forclusión interpersonal $(\mathrm{F}(1,217)=7.337, p<.01$, con $M=21.6$ para colegio privado y $M=18.7$ para colegio público) y a nivel de realización interpersonal $(F(1,216)=9.469, p<.01$, con $M=35.9$ para colegio público y $M=33.3$ para colegio privado), de tal forma que los estudiantes de colegio privado puntúan más alto en forclusión que los estudiantes de colegio público y los estudiantes de colegio público puntúan más alto en realización que los estudiantes del colegio privado.

La estima de sí fue medida con la escala de autoestima de Rosenberg (1973) que fue adaptada para Costa Rica por Smith (2003). Es un instrumento de 10 ítems que fue creado para estudiar la

DIFERENCIAS DE MEDIAS ANOVA POR SEXO Y POSICIONES INTERPERSONALES

\begin{tabular}{lccc}
\hline & & SEXO & \\
\hline $\mathrm{N}=219$ & ANOVA & VALOR DE $p$ & POTENCIA \\
\hline Difusión interpersonal & $F(1,205)=2.356$ & $p=.126$ & $\mathrm{P}=0.333$ \\
Forclusión interpersonal & $F(1,205)=2.711$ & $p=.101$ & $\mathrm{P}=0.374$ \\
Moratoria interpersonal & $F(1,205)=10.960$ & $p<.01$ & $\mathrm{P}=.909$ \\
Realización interpersonal & $F(1,205)=6.022$ & $p=.015$ & $\mathrm{P}=.685$ \\
\hline
\end{tabular}

Elaborado por el autor. 
CUADRO 2

DIFERENCIA DE MEDIAS ANOVA POR COLEGIO Y POSICIONES INTERPERSONALES

\begin{tabular}{lccc}
\hline & & COLEGIO & \\
\hline $\mathrm{N}=219$ & ANOVA & VALOR DE $p$ & POTENCIA \\
\hline Difusión interpersonal & $F(1,217)=0.569$ & $p=.451$ & $\mathrm{P}=.117$ \\
Forclusión interpersonal & $F(1,217)=7.337$ & $p<.01$ & $\mathrm{P}=.769$ \\
Moratoria interpersonal & $F(1,217)=2.415$ & $p=.122$ & $\mathrm{P}=.346$ \\
Realización interpersonal & $F(1,216)=9.469$ & $p<.01$ & $\mathrm{P}=.865$ \\
\hline
\end{tabular}

Elaborado por el autor.

relación de clases sociales y subjetividad. Reportó un índice Alfa de Cronbach de .72. A continuación, su diferencia de medias ANOVA por sexo y tipo de colegio.
El cuadro 3 muestra que no hay relación significativa de la estima de sí con el sexo o el tipo de colegio. A continuación las actividades realizadas con mayor frecuencia en el tiempo libre en ambos colegios.

CUADRO 3

DIFERENCIA DE MEDIAS ANOVA DE ESTIMA DE SÍ PARA SEXO Y TIPO DE COLEGIO

\begin{tabular}{lccc}
\hline & & ESTIMA DE SÍ & \\
\hline ANOVA & VALOR DE $p$ & POTENCIA \\
\hline Sexo $(\mathrm{N}=207)$ & $F(1,205)=3.257$ & $p=.073$ & $\mathrm{P}=.435$ \\
Colegio $(\mathrm{N}=219)$ & $F(1,217)=1.621$ & $p=.204$ & $\mathrm{P}=.245$ \\
\hline
\end{tabular}

Elaborado por el autor.

El cuadro 4 señala que "oír música" (63\%) es la actividad que más realizan los jóvenes del colegio público, frente a "estar con amigos" $(72,2 \%)$ en el colegio privado. Las diferencias más importantes se dan en: el uso de computadora (privado $56,7 \%$ y público 17,3\%) y ayudar en la casa (público $44,2 \%$ y privado $11,1 \%)$. Además, participar en grupos religiosos presenta una diferencia (público $24,6 \%$ y privado $11,1 \%$ ) de forma tal que los jóvenes del colegio público participan más en grupos religiosos que los jóvenes del colegio privado. Con el fin de profundizar en este último resultado, la diferencia de medias ANOVA de la participación en grupos religiosos por sexo y tipo colegio.

El cuadro 5 muestra cómo hay una diferencia significativa del colegio $(F(1,217)$ $=13.022, \mathrm{p}<.01$ ), con $\mathrm{M}=2.5$ para colegio público y $\mathrm{M}=2.1$ para colegio privado) en la participación en grupos religiosos. De forma que los estudiantes del colegio público participan más en grupos religiosos que los estudiantes del colegio privado. 


\section{CUADRO 4 \\ ACTIVIDADES REALIZADAS CON MAYOR FRECUENCIA EN AMBOS COLEGIOS}

\begin{tabular}{clclc}
\hline \multirow{2}{*}{ N=219 } & \multicolumn{2}{c}{ COLEGIO PÚBLICO N=138 } & \multicolumn{2}{c}{ COLEGIO PRIVADO N=81 } \\
\hline PREFERENCIA & ACTIVIDAD & $\%$ & ACTIVIDAD & $\%$ \\
\hline 1 & Oír música & 63 & Estar con amigos & 72,2 \\
2 & Ayudar en la casa & 44,2 & Oír música & 64,1 \\
3 & Estar con la familia & 43,4 & Usar la computadora & 56,7 \\
4 & Estar con amigos & 42,7 & Practicar deportes & 49,3 \\
5 & Descansar & 39,1 & Descansar & 45,6 \\
6 & Ver televisión & 36,9 & Ver televisión & 44,4 \\
7 & Practicar deportes & 24,6 & Estar con la familia & 33,3 \\
8 & Participar en grupos religiosos & 24,6 & Realizar actividades artísticas & 17,2 \\
9 & Estar con el novio o novia & 18,8 & Ayudar en la casa & 11,1 \\
10 & Usar la computadora & 17,3 & Leer & 9,8 \\
11 & Realizar actividades artísticas & 9,4 & Participar en grupos religiosos & 9,8 \\
12 & Leer & 6,5 & Estar con el novio o novia & 6,1 \\
13 & Ir al cine & 3,6 & Ir al cine & 2,4 \\
\hline
\end{tabular}

Elaborado por el autor.

CUADRO 5

DIFERENCIA DE MEDIAS ANOVA DE PARTICIPACIÓN EN GRUPOS RELIGIOSOS PARA SEXO Y TIPO DE COLEGIO

\begin{tabular}{lccc}
\hline & \multicolumn{3}{c}{ PARTICIPACIÓN EN GRUPOS RELIGIOSOS } \\
\hline ANOVA & VALOR DE $p$ & POTENCIA \\
\hline Sexo $(\mathrm{N}=207)$ & $F(1,205)=4.231$ & $p=.350$ & $\mathrm{P}=.462$ \\
Colegio $(\mathrm{N}=219)$ & $F(1,217)=13.022$ & $p<.01$ & $\mathrm{P}=.949$ \\
\hline
\end{tabular}

Elaborado por el autor.

\section{DISCUSIÓN}

Por tratarse de una investigación exploratoria, las hipótesis aquí comentadas son descriptivas, de ninguna manera se hace referencia a algún elemento de causalidad. Faltan más investigaciones que validen los resultados encontrados.
El objetivo general del trabajo es conocer cómo la clase social se relaciona con la construcción de la identidad personal en adolescentes. La evidencia apoya la hipótesis de que la clase social se relaciona con la identidad personal de forma tal que en la dimensión interpersonal los adolescentes de clase trabajadora promedian más alto en la posición de realización y 
los jóvenes de clase burguesa promedian más alto en forclusión.

Realización interpersonal consiste en lograr una representación y compromiso propio luego de explorar alternativas sobre lo que se es y lo que se quiere en amistad, pareja, sexualidad y recreación. Forclusión interpersonal consiste en haber incorporado un proyecto predispuesto por los padres $\mathrm{u}$ otras figuras de autoridad sin haber atravesado un período de búsqueda y definición de la identidad personal (Marcia, 1993).

Se conoce que los jóvenes en situación de desventaja económica presentan con más frecuencia emociones negativas sobre los padres que los jóvenes en mejor posición (Smetana, Campione-Barr y Metzger, 2006). Los padres son quienes generan mayor influencia en las decisiones interpersonales de los hijos, $y$ son un modelo del nivel de satisfacción que ellos tienen de sí mismos (Archer, 1993).

Se hipotetiza que la razón de esta diferencia se encuentra en que los jóvenes del colegio público se han visto expuestos a decidir por sí mismos qué tipo de relaciones interpersonales desean y los jóvenes del colegio privado no. Al igual que lo había encontrado Coté (1996, 2002) en otras investigaciones.

En los jóvenes del colegio público no hay una identificación muy grande con los padres, quienes tampoco les ofrecen un espacio social protegido del estrés cotidiano de las clases populares (Arnold y Doctoroff, 2003) ni de la interacción con diferentes personas y ambientes, como sí sucede con el espacio social que los padres de los jóvenes del colegio privado le construyen a sus hijos.

De esta manera, el adolescente del colegio público no se identifica totalmente con sus padres (Smetana, et ál., 2006) ni con la mayoría de adolescentes de su espacio social que ya están fuera del sistema educativo (Estado de la Nación, 2007). Se ve expuesto por su clase social a decidir por sí mismo qué quiere y qué no. La interacción en su espacio social lo hace cuestionarse, buscar alternativas y decidir qué tipo de relaciones interpersonales desea construir, lo cual no sucede con los jóvenes del colegio privado.

Los jóvenes del colegio privado viven en un mundo creado $y$ protegido por sus padres, quienes les determinan su espacio social (Bourdieu, 2000). Les escogen el colegio, los transportan y les circunscriben sus espacios de interacción (colegio, centros comerciales, clubes privados, amigos). Los adolescentes no se ven expuestos a interactuar fuera de su clase social $y$ tienen menos presión de su espacio social por escoger sus relaciones interpersonales, las cuáles ya están predeterminadas.

Este mecanismo de aislamiento y protección burguesa sirve para que la clase se mantenga y se reproduzca en una posición hegemónica dentro de la sociedad. Las nuevas generaciones se conservan burguesas a través de los espacios sociales que ya están determinados para ello.

En la dimensión interpersonal, las mujeres puntúan significativamente más alto en moratoria interpersonal $y$ realización interpersonal que los hombres. Este resultado es congruente con un gran número de investigaciones empíricas en otros contextos (Marcia, 1993) y en Costa Rica donde Campos (2005) ya había encontrado esta diferencia por género. Parece ser ya una conclusión bien aceptada que las mujeres avanzan cronológicamente más rápido que los hombres en el proceso de construcción identitaria (Streitmatter, 1988).

Esta diferencia en el desarrollo se puede relacionar con otras diferencias de género que existen en el país, las cuales muestran que los hombres son quiénes desertan en mayor proporción del sistema educativo, paradójicamente ganan mejor $y$ viven menos (Estado de la Nación, 2007). Mientras que las mujeres dentro de las familias administran en mayor porcentaje el dinero familiar, establecen las reglas del hogar, se gradúan más del sistema educativo y empiezan a tener relaciones sexuales posteriormente (Consejo Nacional de la Política Pública de la Persona Joven, 2008).

Martínez (2008) señala como con la crisis del capitalismo y la destrucción del estado benefactor el peso de los hogares recae sobre todo en las mujeres, quiénes sostienen sus familias con una sobrecarga cada vez mayor de trabajo no remunerado. Las mujeres dentro del patriarcado podrían tener definido más temprano que tienen que hacer $y$ los hombres gozar de un poco más de tiempo para buscarlo. 
Sin embargo, la relación entre el avance más rápido en el desarrollo y construcción de la identidad personal en las mujeres y las diferencias de género en una cultura patriarcal-capitalista como la costarricense merecen especial atención y futuras investigaciones.

En la estima de sí, no se encontraron diferencias por clase social ni género. La teoría (Melendo, 2006) y diferentes investigaciones internacionales (Smith y Allik, 2005) y costarricenses (Smith, 2003) muestran una influencia de la clase social en la estima de sí del adolescente. Sin embargo, la incidencia es más evidente a medida que las personas van avanzando en la trayectoria de su ciclo vital (O' Rand, 1996).

Si bien es cierto, la clase social incide con más peso entre más edad tengan las personas (Mc Mullin y Cairney, 2004), se espera que ya a los 16 ó 17 años estas diferencias sean notables (Demo y Savin-Williams, 1983). En este caso no lo son. Existe una paridad en los niveles de estima de sí reportados por los jóvenes del colegio público y privado y entre mujeres $y$ hombres.

La autoestima es un proceso dialéctico que se construye en relación y con otros (Melendo, 2006). La respuesta al por qué no hay diferencias puede estar en saber quiénes son "los otros" de estos muchachos, en saber dónde colocan su mirada para construirse.

Para los jóvenes del colegio público el hecho de estar al final del ciclo educativo los coloca en un lugar privilegiado dentro de su espacio social. Están por encima del promedio de educación recibido por sus padres y del resto de jóvenes de su clase social, los cuales en la mayoría de los casos fueron expulsados del sistema educativo. Bajo estas condiciones, ya triunfaron y su sentimiento de valía es igual que el de los jóvenes del colegio privado de quienes a su edad no se espera menos que estar en el colegio.

Para los muchachos del colegio público la familia puede jugar un papel importante en la construcción de su autoestima. Se han encontrado evidencias de casos de jóvenes con pocas condiciones económicas y éxito académico, en los cuales el soporte familiar de sus aspiraciones juega una posición fundamental.
Los jóvenes encuentran en el colegio un sostén al estrés cotidiano. Además, su autopercepción como buenos estudiantes los ayuda a triunfar (Pollard, 1989). Su mirada debe estar en aquellos de su espacio social que ya fueron expulsados del sistema educativo y en sus padres que no alcanzaron terminar el colegio.

Para los jóvenes del colegio privado, estar en el colegio no es otra cosa que el camino trazado con anterioridad. La deserción en la educación privada es significativamente más baja que en la pública (Estado de la Nación, 2007) y su mirada posiblemente está colocada en sus padres: exitosos económicamente y con altos niveles de educación.

Esta paridad de la estima de sí en poblaciones tan distantes de la adolescencia y en las cuales se espera hallar diferencias se podría deber a las características de los jóvenes que participaron en la investigación. Ambos grupos se encuentran dentro del sistema educativo. Los resultados en poblaciones distintas, por ejemplo jóvenes trabajadores fuera del sistema educativo en relación con jóvenes burgueses, podrían ser diferentes.

Los jóvenes del colegio público participan más en grupos religiosos que los jóvenes del colegio privado. La clase social, como era de esperar, incide en las actividades que los jóvenes realizan en su tiempo libre. Las condiciones materiales determinan el tipo de actividad principal que realizan los seres humanos $y$, por ende, las que realizan en su tiempo libre.

Que los jóvenes del colegio público sean quienes más participen de grupos religiosos no es sorprendente. A los jóvenes burgueses su espacio social les permite realizar otro tipo de actividades: practicar deportes, realizar actividades artísticas y usar la computadora, entre otras. Los jóvenes de familia obrera, por tener menos oportunidades, ven en los grupos religiosos un lugar necesario de socialización.

Además, participar en un grupo religioso es una actividad colectiva. Los jóvenes del colegio privado, como lo señala Alarcón y Rosabal (1993), están más orientados a la individualidad y se identifican más con las figuras de autoridad que con su grupo de pares, en contraste con los jóvenes del colegio público que están más 
orientados a la colectividad y que se identifican más entre sí.

Costa Rica es un país profundamente religioso, la última encuesta nacional de juventud muestra que dentro de la población de 15 a 17 años el 30\% de jóvenes participan de algún grupo religioso, siendo la segunda actividad más realizada después de participar en algún equipo deportivo con un $43 \%$ de muchachos (Consejo Nacional de la Política Pública de la Persona Joven, 2008).

Dentro de la tradición marxista, la religión es vista como un instrumento ideológico que amordaza $y$ embrutece a las clases populares. Con la esperanza de una vida eterna después de la muerte, las personas normalizan e interiorizan las desigualdades y explotaciones a las que se ven expuestas cotidianamente. Además, son sometidas a construcciones ideológicas que no les permiten desentrañar las causas económicas y sociales que hacen que ellos, como clase asalariada, mantengan a través de su explotación las riquezas de la burguesía (Luxemburgo, 1905/2004).

Recientemente, surgen datos que muestran cómo en la clase trabajadora se practican más actividades religiosas (Schieman, Nguyen y Elliot, 2003). Es sabido que la participación de jóvenes en grupos religiosos disminuye la posibilidad de que estos se vean envueltos en actividades riesgosas, así como sirve de aliciente para la estima de sí (Sinha, Cnaan, y Gelles, 2007). También se conoce que las creencias religiosas inciden en las actividades y decisiones que las personas realizan respecto a sus ingresos económicos y composición familiar (Lehrer, 2004).

Dentro del paradigma de identidad personal se explica de diferentes maneras la participación de los jóvenes en grupos religiosos. En algunos refleja compromiso, en otros, una falta de interés personal en la religión. Dentro del compromiso algunos jóvenes aceptan la fe desde una edad temprana y la incorporan como un elemento importante en su vida, les permite distinguir lo bueno de lo malo y les deja una buena sensación después de participar en actividades religiosas. Para los no comprometidos, pueden asistir debido a la presión de sus padres o por la oportunidad de interactuar con amigos y pareja (Marcia, 1993).
Sin embargo, que los jóvenes del colegio público sean quiénes más participen en grupos religiosos concuerda con las características de su espacio social. A corto plazo puede fungir como un espacio sano de socialización que los impulsa a seguir estudiando y a no envolverse en actividades de riesgo. A mediano plazo, la historia muestra que les imposibilita cuestionarse el sistema económico vigente.

La tropicalización de los métodos de acceso al conocimiento y producción académica, educativa e ideológica siguen siendo una tarea pendiente para las ciencias sociales de la región. Este trabajo intenta aportar en este sentido. En la investigación se adaptaron instrumentos que han sido validados en múltiples y diferentes contextos (Adams, et ál., 2006) para conocer el desarrollo de la identidad personal en jóvenes adolescentes costarricenses.

Hace más de dos décadas, Marcia (1993) discutía sobre las dificultades de este proceso en espacios no norteamericanos y otros trabajos han apuntado la misma dificultad (Lonner y Berry, 1986). Además, no es el único paradigma que estudia la identidad personal ni la única metodología posible para su abordaje. Con esta dificultad y con los resultados hallados, hay todavía camino por delante.

Comparar cómo la clase social incide en la identidad personal es aportar en el conocimiento y análisis del marco ideológico que valida un sistema económico esquizofrenizante como el capitalista, el cual valida que un grupo social explote $y$ viva de otro.

\section{BIBLIOGRAFÍA}

Adams, Gerald. "The objective measure of egoidentity status: A reference manual". Ontario: University of Guelph. 1998. [Versión electrónica].

Alarcón, Marcela y Rosabal, Mariano. "Conciencia moral e identidad del Yo. Estudio de casos en dos grupos de adolescentes de diferentes clases sociales. Una propuesta teórico metodológica". [Tesis de licenciatura en psicología]. Universidad de Costa Rica, 1993. 
Antunes, Ricardo. "Los nuevos proletarios del mundo en el cambio de siglo". Marxismo Vivo I. Instituto José Luís e Rosa Sundermman. 2000: 112-135.

Archer, S. "Identity status in early and middle adolescents: Scoring criteria". J. Marcia, A. Waterman, D. Matteson, S. Archer y J. Orlofsky. Ego identity: A handbook for psychosocial research. Nueva York: Springer-Verlag, 1993.

Arnold, D. y Doctoroff, G. "The early education of socioeconomically disadvantagen children". Annual Review of Psychology 54. 2003: 517-45.

Berzonsky, Michael. "Identity Style Inventory (Revised Version)". Cortland: State University of New York. 1992. [Versión electrónica].

Bourdieu, Pierre. Poder, derecho y clases sociales. Bilbao: Desclée, 2000.

Campos, Diana. "Las orientaciones culturales en relación con el apego hacia los pares $y$ la identidad personal en adolescentes de 15 a 17 años del cantón de Valverde Vega: contribución al estudio de la correspondencia entre la afectividad y la cultura". [Tesis de licenciatura en psicología]. Universidad de Costa Rica, 2005.

Cardoso, F. y Pérez, H. El concepto de clases sociales: bases para una discusión. San José: Nueva Década, 1982.

Castro, Ricardo. "Estilos y figuras de apego predominantes en la adolescencia: estimación de la validez y la coherencia interna del "Cuestionario de estilos de apego" en el contexto costarricense". [Tesis de licenciatura en psicología]. Universidad de Costa Rica, 2006.

Consejo Nacional de la Política Pública de la Persona Joven. Primera Encuesta
Nacional de Juventud. San José: UNFPA, 2008.

Coté, James. "Sociological perspectives on identity formation: the culture-identity link and identity capital". Journal of Adolescence 19. 1996: 417-428.

Coté, James. "The role of identity capital in the transition to adulthood: The individualization thesis examined". Journal of Youth Studies 5 (2). 2002:117134.

Demo, D. y Savin-Williams, R. "Early adolescent self-esteem as a function of social class: Rosengerg and Pearlin revisited". The American Journal of Sociology (88) 4. University of Chicago. 1983: 763-774.

Instituto Nacional de Estadísticas y Censos. Encuesta de Hogares. 2006. En: <http:// www.inec.co.cr $>$ [consultado 4 de octubre 2006].

Erikson, Erik. Sociedad y Adolescencia. Buenos Aires: Editorial Paidós, 1927.

Frable, D. "Gender, racial, ethnic, sexual, and class identities". Annual Review of Psychology 48. 1997: 139-162.

Lehrer, E. "Religion as a determinant of economic and demographic behavior in the United States". Population and Developmental Review 30 (4). 2004: 707726.

Lott, Bernice. "Cognitive and behavioral distancing from the poor". American Psychologist 57 (2). 2002: 100-110.

Lonner, W. y Berry, J. Field methods in crosscultural research. California: Sage, 1986.

Luxemburgo, Rosa (1905). "El socialismo y las iglesias". Marxismo Vivo 11. Instituto 
José Luís e Rosa Sundermman. 2004: 112-127.

Marcia, James. "The ego identity status. Approach to ego identity". Marcia, J., Waterman, A., Matteson, D., Archer, S. y Orlofsky, J. Ego identity: A handbook for psychosocial research. Nueva York: Springer-Verlag, 1993.

Marcia, James. "Adolescent, identity, and the bernardote family". Identity: An International Journal of Theory and Research 2 (3). Psychology Press. 2002: 199-209.

Martín-Baró, Ignacio (1983). Acción e ideología. Décima edición. El Salvador: UCA Editores, 2004.

Martínez, Juliana. Domesticar la incertidumbre en América Latina. Costa Rica: Editorial de la Universidad de Costa Rica, 2008.

Mc Mullin, J. y Cairney, J. "Self-esteem and the intersection of age, class, and gender". Journal of Aging Studies 18. 2004: 75-90.

Melendo, T. Felicidad y autoestima. Madrid: Ediciones Internacionales Universitarias, 2006.

MIDEPLAN. Distritos prioritarios para el gobierno. Costa Rica: Gobierno Central, 2005.

Miliban, R. "Análisis de clases". El sistema mundo, las clases y la cultura. Cuadernos de Ciencias Sociales. Costa Rica: FlACSO, 1996.

O'Rand, A. "The precious and the precocious: Understanding cumulative disadvantage and cumulative advantage over the life course". Gerontologist 36. Gerontological Society of America. 1996: 230-238.

Pérez, Rolando. "Dimensiones psicosociales al uso de tecnologías de la información y la comunicación en estudiantes de secundaria". [Informe final actividad de investigación: 723-A6-707]. Universidad de Costa Rica: Instituto de Investigaciones Psicológicas, 2006.

Pollard, D. "Against the odds: A profile of academic achievers from the urban underclass". The Journal of Negro Education 58 (3). Howard University. 1989: 297-308.

Programa estado de la nación en desarrollo humano sostenible. Duodécimo informe estado de la nación en desarrollo humano sostenible. San José, c R. 2006.

Programa estado de la nación en desarrollo humano sostenible. Decimotercer informe estado de la nación en desarrollo humano sostenible. San José, c R. 2007.

Rosenberg, M. La autoimagen del adolescente y la sociedad. Buenos Aires: Editorial Paidós, 1973.

Schieman, S., Nguyen, K. y Elliot, D. "Religiosity, socioeconomic status, and the sense of mastery". Social Psychology Quarterly 66 (3). American Sociological Association. 2003: 202-221.

Schwartz, S. "The evolution of eriksonian and neo-eriksonian identity theory and research: A review and integration". Identity: An international journal of theory and research (1) 1. Psychology Press. 2001: 7-58.

Sinha, J., Cnaan, R. y Gelles, R. "Adolescent risk behaviors and religion: findings from a nacional study". Journal of Adolescence 30. 2007: 231-249.

Smetana, J., Campione-Barr, N. y Metzger, N. "Adolescent development in interpersonal and societal contexts". Annual Review of Psychology 57. 2006: 255-84. 
Smith, Vanessa. Acculturation and psychological adaptation. Connecticut: Greenwood Press, 2003.

Streittmater, J. "Ethnicity as a mediating variable of early adolescent identity development". Journal of Adolescence 11. 1989: 335-346.
Schmitt, D. y Allik, J. "Simultaneous administration of the Rosenberg selfesteem scale in 53 nations: exploring the universal and culture-specific features of global self-esteem". Journal of Personality and Social Psychology 89 (4). American Psychological Association. 2005: 623-642. 